\title{
Editor's Note: Special Issue on Novel Representations and Learning Methods in Computer Vision
}

๑) Springer Science+Business Media, LLC, part of Springer Nature 2018

International Journal of Computer Vision gratefully acknowledges the editorial work of the scholars listed below on the special issue entitled "Novel Representations and Learning Methods in Computer Vision”.

\author{
Bastian Leibe \\ RWTH Aachen \\ Germany \\ leibe@vision.rwth-aachen.de \\ Jiri Matas \\ Czech Technical University, Prague \\ Czech Republic \\ matas@cmp.felk.cvut.cz \\ Nicu Sebe \\ University of Trento \\ Italy \\ sebe@ disi.unitn.it \\ Max Welling \\ University of Amsterdam \\ Netherlands \\ m.welling@uva.nl
}

Publisher's Note Springer Nature remains neutral with regard to jurisdictional claims in published maps and institutional affiliations. 